\title{
III Traditionelle Funktionen des Namens
}

Angesichts des derzeitigen Zustands drängt sich die Frage nach den rechtspolitischen Leitsternen auf, an denen sich der Gesetzgeber bei der Regelung des Namensrechts bisher orientiert hat.

\section{Individualisierung und Klassifizierung des Namensträgers}

Die Funktionen des Namens in Familie und Gesellschaft, die der Gesetzgeber in seinem Namensrecht nutzen kann, sind vielfältig und potentiell widersprüchlich. ${ }^{32}$ Der Name einer natürlichen Person erfüllt traditionell vor allem zwei Hauptzwecke:

Zum einen ermöglicht der Name eine sprachliche Individualisierung eines Menschen. Die Gesellschaft benennt ihre Mitglieder, um sie von anderen unterscheidbar zu machen, vor allem in der sprachlichen Kommunikation.

Zum anderen kann ein Name zur Klassifizierung der Gesellschaftsmitglieder dienen und sprachlich kodiert Auskunft über deren Eigenschaften geben, etwa im Hinblick auf das Geschlecht (weiblicher oder männlicher Vorname), die Zugehörigkeit zu einer Gruppe, vor allem zu einer Familie beispielsweise kraft Abstammung oder Eheschließung (Familienname), die gesellschaftliche Stellung dieser Gruppe (ehemalige Adelsprädikate, bürgerlicher Name) oder die Funktion des Namensträgers in dieser Gruppe, etwa als Familienoberhaupt („Fürst“ als Bezeichnung des jeweiligen Chefs eines Adelshauses), aber auch die Abstammung von einer Person (Vatersname). Der Wortsinn des Namens hat seine Klassifizierungsfunktion dagegen weitgehend verloren. Eine Frau Müller wird heutzutage keine Mühle mehr betreiben und ein Herr Schmitz nicht im metallverarbeitenden Gewerbe tätig sein. Eine Gesellschaft kann selbstredend in der sprachlichen Kommunikation Mitglieder auch mittels anderer Kennzeichen klassifizieren, die nicht Namensbestandteile sind, wobei die Abgrenzung oftmals nicht einfach ist: Der Klassifizierung des Gesellschaftsmitglieds dienen neben echten Adelsprädikaten in ständischen Gesellschaften auch akademische Grade,

32 Ein Überblick über die im deutschen Schrifttum diskutierten Namensfunktionen findet sich etwa bei Diethelm Klippel Der zivilrechtliche Schutz des Namens (1985) S. 355 ff. und Dietrich Nelle Der Familienname - Perspektiven für eine rechtsvereinheitlichende Reform (Teil I), FamRZ 1990, 809 (809 f.); die von mir im Folgenden als Individualisierung und Klassifizierung sowie unten (V.1.) als Selbstdarstellung bezeichneten Namensfunktionen ziehen lediglich die Grenzen und den Abstraktionsgrad der bisher diskutierten Funktionen anders.

Ә OpenAccess. (๑) 2020 Juristische Gesellschaft zu Berlin, publiziert von De Gruyter.

(c) BY-NC-ND Dieses Werk ist lizenziert unter der Creative Commons Attribution-NonCommercial-NoDerivatives 4.0 License. https://doi.org/10.1515/9783110709872-004 
Titel und Würden (etwa „Professor“33, „Doktor“ oder - vor allem in Österreich „Magister“) und Dienst- wie Berufsbezeichnungen (etwa „Richter am Oberlandesgericht“, „Regierungsdirektor“, „Rechtsanwalt“ oder „Notar“).

Freilich ist der Name nicht das einzig denkbare und vor allem nicht das effektivste Mittel zur Individualisierung und Klassifizierung einer Person. Nicht ohne Grund operieren staatliche und private Institutionen mittlerweile vornehmlich mit Personennummern, etwa in Deutschland mit der steuerlichen Identifikationsnummer nach $\S 139 \mathrm{~b}$ der Abgabenordnung ${ }^{34}$ oder der Rentenversicherungsnummer nach $\S 147$ des Sechsten Buches des Sozialgesetzbuchs ${ }^{35}$. Nummerische Bezeichnungen können Institutionen einmalig und dauerhaft - ja sogar über den Tod der Person hinaus - vergeben (Individualisierung). ${ }^{36}$ Auch können Nummern sehr viel einfacher, präziser und zielgerichteter als ein Name Auskunft über Eigenschaften der Person (Klassifizierung) geben, indem diese Eigenschaften entweder unmittelbar in der Nummer kodiert oder mittelbar in einem Register hinterlegt und mit der Personennummer verknüpft werden. Die Digitalisierung der öffentlichen Register verstärkt diese Überlegenheit von namensunabhängigen Individualisierungs- und Klassifizierungsmitteln wie Personennummern noch einmal. ${ }^{37}$ Aus heutiger Sicht wenig überzeugend sind damit die Gründe für die ersten Kodifikationen des Namensrechts, die den Namen der Person seit der Französischen Revolution von der Sitte in das bürgerliche Recht überführt haben (vgl. noch unten VI.): Die gesetzliche Regelung des Namensrechts etwa im preußischen Allgemeinen Landrecht und im österreichischen Allgemeinen Bürgerlichen Gesetzbuch wird historisch vor allem mit dem Wunsch des Staates erklärt, junge Männer für Zwecke der allgemeinen Wehrpflicht zu erfassen. ${ }^{38}$ Angesichts der Schwächen des Namens als Klassifizierungsmittel

33 Oftmals herrscht Unklarheit über die Natur des Professorentitels. Im Freistaat Bayern handelt es sich nach Art. 12 Abs. 1 S. 1 des Bayerischen Gesetzes über die Rechtsverhältnisse der Hochschullehrer und Hochschullehrerinnen sowie des weiteren wissenschaftlichen und künstlerischen Personals an den Hochschulen vom 23.5.2006, BayGVBl. 2006, 230, bei Professoren im Beamtenverhältnis auf Lebenszeit jedenfalls nach ihrem Eintritt in den Ruhestand beim Professorentitel nicht mehr um eine Dienstbezeichnung, sondern um eine akademische Würde; außerdem dürfen bayerische Professoren der Besoldungsgruppe W 3, allerdings nur an Universitäten und Kunsthochschulen, auch den Titel „Ordinarius“ bzw. „Ordinaria“ führen, vgl. Art. 12 Abs. 3 des Gesetzes, was ebenfalls eine akademische Würde sein dürfte.

34 In der Fassung der Bekanntmachung vom 1.10.2002, BGBl. 2002 I S. 3866.

35 In der Fassung der Bekanntmachung vom 19.2.2002, BGBl. 2002 I S. 754.

36 Dieter Schwab Personenname und Recht, StAZ 2015, 354 (362).

37 Eckpunkte zur Reform des Namensrechts (Fn. 2) S. 4 unter II. 3.

38 Hans Ficker Das Recht des bürgerlichen Namens (1950) S. 139, wonach die allgemeine Wehrpflicht einer der „Schrittmacher“ war. 
greift der Staat heute kaum noch auf die Klassifizierungsfunktion des Namens zurück: Regeln wie die des südkoreanischen Eherechts, die Personen mit dem gleichen Familiennamen als Ausprägung des Eheverbots der Verwandtschaft die Eheschließung verweigert, ${ }^{39}$ musste der Gesetzgeber mittlerweile aufgeben.

Dennoch stand trotz dieser Schwächen der Individualisierungs- und Klassifizierungsfunktion auch im deutschen Namensrecht ursprünglich die Individualisierung und Klassifizierung des Namensträgers als Regelungsziel im Vordergrund:

Zwar verhindert das Namensrecht im Hinblick auf die Individualisierung nicht, dass ein und derselbe Name mehreren Personen zugewiesen wird, aber das deutsche Recht war stets - wenigstens im Ansatz - bestrebt, für eine Namenskontinuität zu sorgen, d.h. für eine grundsätzliche Unabänderlichkeit des Namens. Insbesondere schränkt das eben erwähnte Namensänderungsgesetz bis heute eine isolierte Namensänderung ein und fordert hierzu einen wichtigen Grund (oben II.). Welche illiberalen Wirkungen dieses Gesetz entfaltet, das bei Lichte betrachtet nicht die Namensänderung, sondern ihre Beschränkung zum Gegenstand hat, verdeutlicht ein Blick in die Vergangenheit: Vor der einheitlichen Regelung und Beschränkung der öffentlichrechtlichen Namensänderung durch das Namensänderungsgesetz war es herrschende Meinung, dass die Bürger ihren Vornamen beliebig ändern können, da das Bürgerliche Gesetzbuch hier - anders als bei Änderungen des Familiennamens - keine Grenzen setzt. ${ }^{40}$

Auch war das deutsche Namensrecht lange Zeit darauf gerichtet, mithilfe des Personennamens bestimmte Klassifizierungsmerkmale abzufragen, insbesondere mithilfe des Vornamens das Geschlecht des Namensträgers und mithilfe des Familiennamens seine Familienbeziehungen. Es war noch bis vor Kurzem unbestritten, dass die Eltern dem Kind als Ausfluss ihrer Personensorge nicht nur keine ungebräuchlichen Vornamen geben durften (oben I.). Ferner musste der gewählte Vorname das Kind eindeutig als einem der - damals noch binären (vgl. heute $§ 22$

39 So der alte $\S 809$ Abs. 1 des koreanischen Min beob.

40 Otto Opet Das Namenrecht des Bürgerlichen Gesetzbuchs, AcP 87 (1897) 313 (350): „Die Zulässigkeit der beliebigen Annahme von Vornamen ist nicht unbestritten. Auch hier wollen manche Autoren aus dem Umstand, daß das Personenstandsgesetz keine Bestimmungen über nachträgliche Vornamenseintragung enthalte, darauf schließen, daß als Vornamen nur die bei der Geburtsanzeige oder im Berichtigungsverfahren in das Standesregister eingetragenen Vornamen gebraucht werden dürften; aber wie vorher muss auch hier betont werden, daß das Reichsgesetz nur die Registerführung regelt, das materielle Namenrecht aber in keiner Weise betrifft. Eine Entscheidung kann also nur aus den Landesgesetzen gewonnen werden, so daß die Annahme von Vornamen überall, wo dem kein landesgesetzliches Verbot entgegensteht, dem freien Belieben eines jeden überlassen ist“. 
Abs. 3 und § 45b PStG) - Geschlechter zugehörig kennzeichnen..$^{41}$ Vor allem aber prägte ein dynastisches Denken die Bildung des Familiennamens, das auf vorrepublikanische Ziele des Namensrechts hinweist, nämlich die „Sicherung der ständisch-familiären Ordnung““.42 Die Urfassung des Bürgerlichen Gesetzbuchs leitete den Namen jedenfalls im damaligen Idealzustand der ehelichen Familie von einem Stammvater ab und gab diesen innerhalb einer Dynastie in männlicher Linie an alle in direkter Linie von diesem Stammvater abstammenden Familienmitglieder weiter. Nicht nur die Frau erhielt mit der Eheschließung den Familiennamen des Mannes ( $\$ 1355$ BGB a.F. [1900]), sondern auch die in der Ehe geborenen Kinder erhielten den Namen des Vaters (§ 1616 BGB a.F. [1900]). Allein uneheliche Kinder trugen den Namen ihrer Mutter (§ 1706 Abs. 1 BGB a.F. [1900]).

Individualisierung und Klassifizierung sind widerstreitende Fernziele, die kein Regelgeber in seinem Namensrecht vollständig umzusetzen vermag. So kann die Klassifizierungsfunktion das Individualisierungspotential des Namens einschränken, speziell im Hinblick auf die Namenskontinuität. Immer wenn sich die klassifizierungsrelevanten Eigenschaften ändern, muss sich konsequenterweise auch der Name ändern. Das betrifft vor allem den Familiennamen, soweit dieser über die Familienzugehörigkeit Auskunft geben soll und sich die jeweils maßgebliche Affiliation ändert, z. B. durch Eheschließung oder Beendigung der Ehe, Korrektur der rechtlichen Abstammung, Adoption oder - Stichwort Einbenennung (oben II.) - Aufnahme in eine neue soziale Familie. Da sich - auch aus Gleichbehandlungsgründen (vgl. noch unten IV. 2. a) - die Klassifizierungsfunktion auf das Verhältnis zu einem konkreten Elternteil oder Ehegatten beschränken muss, wird die Namenskontinuität als notwendiges Element einer namensrechtlichen Individualisierung durchbrochen. Besonders der Ehename, seine Wandelbarkeit und seine Fernwirkungen auf den Namen der Kinder stehen bereits seit Langem als Gefahr für die Namenskontinuität und auch das Wohl des Kindes (näher noch unten V. 2. a) in der Kritik. ${ }^{43}$ Aber auch der Vorname kann von der Dynamik persönlicher Merkmale betroffen sein und eine Namenskontinuität und damit Individualisierung des Namensträgers abschwächen. So gestattet das

41 Die ältere Rechtsprechung zur Geschlechtsbezogenheit des Vornamens wird etwa von Reinhard Hepting in: Reinhard Hepting/Berthold Gaaz, Personenstandsrecht mit Eherecht und Internationalem Privatrecht, Band II (Stand 42. Lieferung 2009) Rn. IV-736 ff., dargestellt, einschließlich der ausführlichen Judikatur zur im Einzelfall sehr schwierigen Frage, welche Namen männlich, weiblich und geschlechtsneutral sind.

42 Schwab (Fn. 36) 355.

43 Etwa Michael Coester Fortschritt oder fortgeschrittene Auflösung im Recht des Personennamens, StAZ 1984, 298 (299 ff.); Ingeborg Schwenzer Namensrecht im Überblick - Entwicklung Rechtsvergleich - Analyse, FamRZ 1991, 390 (394 f.). 
deutsche Recht transgeschlechtlichen Personen und - seit Neuestem - auch Personen mit Varianten der Geschlechtsentwicklung eine Änderung des Vornamens, um ihre (neue) Geschlechtsidentität auch namensrechtlich zu dokumentieren ( $\S \S 1,8$ Abs. 2 des Transsexuellengesetzes ${ }^{44}$ und $\S 45 \mathrm{~b}$ Abs. 1 S. 3 PStG). Dennoch dürfte selbst in einem streng auf die Klassifizierung der Namensträger ausgerichteten System die Dynamik des Namens und damit der Widerspruch zwischen Klassifizierung und Individualisierung im Laufe der Geschichte eher abgenommen haben, vor allem wegen des eben geschilderten Verlusts des Namenswortsinns als Klassifizierungsmerkmal. Heute liest man mit Erstaunen im hebräischen Tanach im Buch Rut über die Namensänderung der Noomi, der Urgroßmutter des späteren König David. Noomi verkündet, nachdem sie nach vielen Schicksalsschlägen nach Bethlehem zurückkehrt: „Nennt mich nicht mehr Noomi (Liebliche), sondern Mara (Bittere); denn viel Bitteres hat der Allmächtige mir getan“ ${ }^{45}$ Heute würde eine Noomi (bzw. eher Noemi), auch wenn sie voller Bitterkeit über ihr Schicksal ist, kaum gerade deshalb mit ihrem Vornamen hadern. Ohnehin würde ihr Namensänderungswunsch wegen eines Wandels des subjektiv empfundenen Klassifizierungsmerkmals an den unbarmherzigen Grenzen der öffentlichrechtlichen Namensänderung (oben II.) scheitern, jedenfalls in der Bundesrepublik.

\section{Private oder öffentliche Interessen jenseits der Individualisierung und Klassifizierung?}

Neben der Individualisierungs- und Klassifizierungsfunktion spielen weitere private Interessen oder Ordnungsinteressen des Staates traditionell nur eine untergeordnete Rolle bei der Ausgestaltung des Namensrechts.

Der Wahrung von Privatinteressen ist etwa die Kindeswohlprüfung bei der Vornamensgebung (oben I.) oder die Möglichkeit der öffentlichrechtlichen Namensänderung aus wichtigem Grund (oben II.) geschuldet. Der rechtlich zugewiesene Name darf nicht das Persönlichkeitsrecht des Namensträgers verletzen.

Bei der Ausgestaltung des Namensrechts haben bisher aber auch vereinzelt Ordnungsinteressen des Staates Einfluss genommen. So begrenzt das deutsche Namensrecht beispielsweise mehrgliedrige Namen. Zwar besteht keine Silbenhöchstanzahl für die verschiedenen Namensbestandteile; eine solche Grenze

44 Gesetz über die Änderung der Vornamen und die Feststellung der Geschlechtszugehörigkeit in besonderen Fällen vom 10.9.1980, BGBl. 1980 I S. 1654.

45 Rut 1:20 (Einheitsübersetzung). 
müsste unmittelbar in existierende Namen eingreifen. Aber bei der Namensbestimmung achtet das Recht darauf, dass die Namensbestandteile nicht überhandnehmen. So ist es bei der Vornamensbestimmung (oben I.) anerkannt, dass die Eltern dem Kind nicht eine unbegrenzte Anzahl von Vornamen geben können. Dieses Verbot lässt sich mit Kindeswohlgesichtspunkten nur schwer rechtfertigen (kein Kind muss alle Vornamen im Alltag führen), sondern vor allem mit dem Ordnungsinteresse des Staates an einer ordnungsgemäßen Register- und Urkundenführung. ${ }^{46}$ Aber auch beim Familiennamen nimmt der Gesetzgeber die Länge des Namens in den Blick, speziell durch das Verbot eines Begleitnamens (dazu sogleich näher IV. 2. a) bei mehrgliedrigen Namen (§ 1355 Abs. 4 S. 2 BGB), um mehr als zweigliedrige Namen (z. B. „Müller-Meier-Schmitz“) zu verhindern. Zwar scheint das Bundesverfassungsgericht, das gegen dieses Verbot verfassungsrechtlich nichts einzuwenden hatte, den Zweck dieses Verbots eher in einer Wahrung der Individualisierungsfunktion (oben III. 1.) zu sehen: Unbegrenzte Namensketten nähmen dem Namen seine identifikationsstiftende Kraft. ${ }^{47}$ Allerdings überzeugt diese Argumentation wenig: Je mehr Bestandteile der Familienname einer Person enthält, desto einmaliger ist der Name und desto besser dient er der Individualisierung seines Trägers. Das Gleiche gilt im Übrigen auch für die Klassifizierungsfunktion: Mehrgliedrige Familiennamen geben sehr viel umfangreicher über die Familienzugehörigkeiten des Namensträgers Auskunft als eingliedrige Namen. Plausibler ist daher die Erwägung des Gesetzgebers, dass mehrgliedrige Namensketten den Rechts- und Geschäftsverkehr unnötig belasten, ${ }^{48}$ womit klassische Ordnungsinteressen angesprochen werden.

46 Näher zu Inhalt und Begründung des Verbots Hepting/Dutta (Fn. 6) Rn. IV-439 ff.

47 BVerfG 5.5.2009, BVerfGE 123, 90 = StAZ 2009, 179 = FamRZ 2009, 939 (941, 942) m. kritischer Anm. Henrich; so bereits BVerfG 30.1.2002, BVerfGE 104, 373 = StAZ 2002, 72 = FamRZ 2002, 306 (309).

48 Siehe z.B. die Stellungnahme des Bundesrats zum Ersten Gesetz zur Reform des Ehe- und Familienrechts (1. EheRG), BT-Drucks. 7/650, S. 257; Begründung des Entwurfs eines Gesetzes zur Neuordnung des Familiennamensrechts, BT-Drucks. 12/3163, S. 15 f. 